\title{
Bicuspidization of a Mandibular First Molar With Furcation Defect: A Case Report
}

\author{
Ferdina Nidyasari ${ }^{1}$, Wignyo Hadriyanto ${ }^{2}$, Margareta Rinastiti ${ }^{2}$ \\ ${ }^{1}$ Post Graduate Student of Conservative Dentistry Specialistic Program, Faculty of Dentistry, \\ Universitas Gadjah Mada, Yogyakarta \\ ${ }^{2}$ Department of Conservative Dentistry, Faculty of Dentistry, \\ Universitas Gadjah Mada, Yogyakarta \\ *Coresponding author email : ferdinanidyasari1985@gmail.com \\ Authorsemail :wignyodentistugm@gmail.com, rinastiti@ugm.ac.id
}

\begin{abstract}
The increased desire of patients to maintain their dentition has forced conservative dentistry to conserve the teeth which are planned to be removed. Defect in furcation area resulting from the lesion has always been a concern regarding the choice of treatment. Bicuspidization is a process to separate distal and mesial roots in mandibular molars along with their coronal portions into two segments that retain individually. The purpose of this case report is to inform about bicuspidization treatment to preserve the tooth. A 19 year old male came to Prof. Soedomo Dental Hospital complained of swelling in left mandibular posterior region since 1 month ago. Clinical examination showed temporary restoration on occlusal area of 36 tooth, sensitive to percussion, grade 1 mobility, probing depth $7 \mathrm{~mm}$ in mid buccal aspect, vitality test yielded no response, and there was a chronic apical abscess. Dental history revealed patient had dental filling 2 years ago. Periapical radiograph confirmed Class II furcation involvement with vertical bone loss and radiolucent area in mesial and distal periapical. Bone support for both roots remain intact. Based on clinical examination, bicuspidization followed by porcelain fused to metal restoration would be performed. Conclusion: Bicuspidization can be considered as suitable alternative treatment to extraction for multirooted teeth with Class II furcation involvement. Through this procedure, unfavorable anatomic and furcation defects were removed, tooth structure can be preserved and good healing response is achievable.
\end{abstract}

Keywords: furcation defect, bicuspidization, porcelain fused to metal restoration, Prof. Soedomo Dental Hospital 


\section{INTRODUCTION}

The increased desire of patients to maintain their dentition has forced conservative dentistry to conserve the teeth which are planned to be removed [1]. Defect in furcation area resulting from the lesion has always been a concern regarding the choice of treatment. Furcation involvement can be defined as the loss of attachment and radiographic evidence of bone loss in the bifurcation and trifurcation areas of multi-rooted teeth. The furcation defects vary from a subtle loss of attachment in the buccal furcation area, forming a shallow pocket, to advanced pathology with deep pockets $>10 \mathrm{~mm}$, advanced bone loss and clinical exposure of the furcation [2].

The advanced teeth conservation treatment like endodontic surgery intend to maintain the teeth so that they can be in good condition for a long time. Tooth separation and resection procedures are used to preserve as much tooth structure as possible rather than sacrificing the whole tooth. In some cases, the molar with severe damage on bifurcation can be maintained by endodontic surgery treatment withbicuspidization procedure [3]. Bicuspidization is the separation of mesial and distal roots of mandibular molars along with their coronal portion, where both segments are then retained individually [4].

This case report describes a treatment procedure for mandibular molar with furcation involvement that includes endodontic surgery with bicuspidization and then the tooth was restored with porcelain fused to metal restoration.

\section{CASE REPORT}

A 19 year old male came to Prof. Soedomo Dental Hospital complained of swelling in left mandibular posterior region since 1 month ago. Clinical examination showed temporary restoration on occlusal area of 36 tooth, sensitive to percussion, grade 1 mobility, probing depth $7 \mathrm{~mm}$ in mid buccal aspect, vitality test yielded no response, and there was a chronic apical abscess (Figure. 1). Dental history revealed patient had dental filling 2 years ago. Periapical radiograph confirmed Class II furcation involvement with vertical bone loss and radiolucent area in mesial and distal periapical. Bone support for both roots remain intact (Figure 2). Based on clinical examination, bicuspidization followed by porcelain fused to metal restoration would be performed.

Root canal treatment was performed first before bicuspidization. One month after obturation, bicuspidization procedure was performed under local anesthesia with a full thickness flap which reflected extending from the distal surface of 34 to the mesial surface of 37 (Figure 3). Upon reflection of the flap, curettage was performed to remove chronic inflammatory tissues. The tooth was separated vertically using long shank fissure carbide bur into mesial and distal portion (Figure 4). The furcation area was trimmed to ensure that no residual debris were present that could cause further periodontal irritation, followed by bone graft placement along with the membrane (Figure 5 and 6). The working area was irrigated adequately with sterile saline. The flap was repositioned and suture were placed between mesial, distal and mid-portion of the separated tooth (Figure 7). Bicuspidization procedure confirmed with periapical radiograph (Figure 8). Systemic antibiotics, analgesics and anti-inflammatory were prescribed and post operative instructions were given.

Patient was recalled after ten days for suture removal, and followed by crown preparation and fiber post inserted on two separated halves of 36 one week later. Impression was recorded and temporization done. After two months, porcelain fused to metal crowns were cemented on mesial and distal of the tooth (Figure 9). Three months follow up after bicuspidization showed good healing response on furcation and periapical area. Radiographic evaluation had been taken and an increased radio-opacity had shown in furcation area (Figure 10)

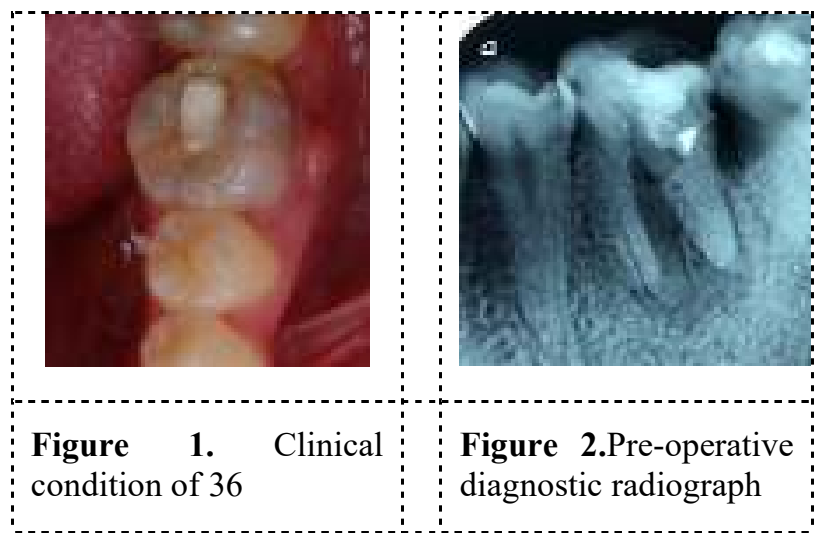




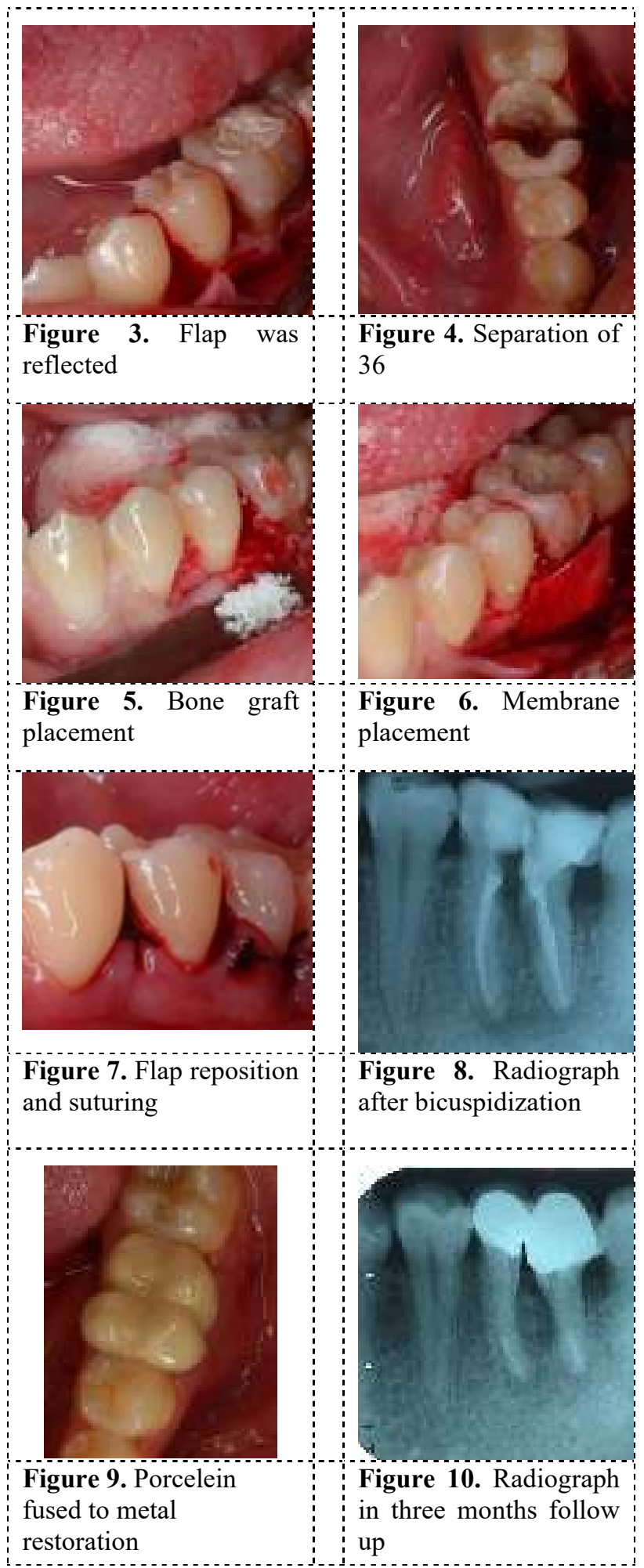

\section{DISCUSSION}

Based on the present case report, patient complained of pain and periapical radiograph showed Class II furcation involvement with vertical bone loss and radiolucent area in mesial and distal periapical, bone support for both roots remain intact. Radiolucent area in mesial and periapical resulted from pulpal disease while the bone loss became an outcome of periodontal disease.

Bicuspidization is one of the alternative to avoid tooth extraction. This procedure represents a form of conservative dentistry which aims to retain as much of the original tooth structure as possible [5]. Bicuspidization is defined as splitting the mandibular molar vertically through the furcation and leaving two separate roots that will be treated as bicuspids [6]. This technique is indicated in Class II and III furcation involvements. This procedure is able to remove unfavorable anatomic contours, hemiseptal defects, and deep intra-bony defects. This procedure will also allow the separated segments to bear the occlusal load adequately for long term preservation [7]. Farshchian and Kaiser has reported the success of a molar bicuspidization depends on three factors [8]:

1. Bone support individual tooth sections must adequate and stable

2. Absence of severe root fluting of the distal aspect of the mesial root or mesial aspect of the distal root.

3. Adequate separation of the mesial and distal roots, to enable the creation of an acceptable embrasure for effective oral hygiene

In this case, since both the mesial and distal roots of the tooth still have an adequate bone support, it was decided to perform bicuspidization procedure with bone graft placement to treat the osseous defect. Root canal treatment was performed first before bicuspidization to treat the pulpal disease. After bicuspidization procedure, the tooth was restored with porcelain fused to metal restoration with fiber post. Fiber post has good retention properties under mechanical strain as a result of their low elastic modulus, which is similar to that of dentine. This biomechanical behaviour avoids root fractures [9]. Porcelain fused to metal 
chosen as post bicuspidization restoration because it has good mechanical properties and satisfactory esthetic results along with a clinically acceptable quality of their marginal and internal adaptation [10].

Three months follow up showed good healing response on furcation and periapical area. Radiographic evaluation has been taken and an increased radio-opacity had shown in furcation area. Compared to intial radiograph, three months postoperative radiograph showed better bone healing improvement after bicuspidization and placement of bone graft along with the membrane. Bone grafts are used as a filler and scaffold to facilitate bone formation and promote wound healing. These grafts are bioresorbable and have no antigen-antibody reaction. These bone grafts act as a mineral reservoir which induces new bone formation [11]. This case used demineralized bone xenograft granule or BX-G (Batan) as bone grafting material. Xenografts consist of bone tissue taken from a different species such as bovine, and have osteoconductive properties and preserve the original bone mineral structure, which is more complex than that of synthetic materials [12]. A bovine derived xenogenic bone graft (BDX) has been extensively used with positive clinical results over the last decade in the treatment of periodontal infrabony and furcation defects, alone or in combination with membranes or enamel matrix derivatives [13]. Periodontal regeneration by membrane techniques is based on the principal of separation of different tissues by surgical placement of physical barriers. Barrier membranes allows for defect space to be maintained for regenerating tissues which would otherwise be infiltrated and occupied by the epithelial cells. If used in combination with bone grafts, then the membranes also serve to stabilize, contain and preserve the graft materials. This also results in reducing the rate of graft resorption [14].

\section{CONCLUSION}

Bicuspidization can be considered as suitable alternative treatment to extraction for multirooted teeth with Class II furcation involvement.
Through this procedure, unfavorable anatomic and furcation defects were removed, tooth structure can be preserved and good healing response is achievable.

\section{REFERENCES}

[1] Mantri V, Maria R, Kamat S, Raut AW, 2013, Root Amputation: Case Reports and Review, Endodontology; 25(2):89-95

[2] Abu-Hussein M, Watted N, Abdulgani A, 2015, Bicuspidization of Mandibular Molar; A Clinical Review; Case Report, IOSR Journal of Dental and Medical Sciences (IOSR-JDMS); 14(6): 77-85

[3] Vandersall DC and Detamore RJ, 2002. The Mandibular Molar Class III Furcation invasion. A Review of Treatment Options, $J A D A$; 133(1): 55-60

[4] Arabaci T, Çiçek Y, Güngör H, 2012, Treatment of a Complicated Iatrogenic Furcation Perforation in a Mandibular Molar with Bicuspidization Technique: A Case Report, $M \ddot{U} S B E D ; 2(3): 130-3$

[5] Nikita T, Manwar NU, Chandak M, 2011, Bicuspidization - A Case Report and Review. Case Study, Case Rep; 1:187-91

[6] Augsburge RA, 1976, Root Amputations and Hemisections, Gen Dent; 24:35-8

[7] Park SY, Shin SY, Yang SM, Kye,SB, 2009, Factors Influencing The Outcome of Root Resection Therapy in Molars: A 10-Year Retrospective Study, J Periodontol; 80:3240

[8] Farshchian F, Kaiser DA. 1988, Restoration of The Split Molar: Bicuspidization, Am J Dent; $1: 21-2$

[9] Bru E, Forner L, Llena C, Almenar A, 2013 , Fibre Post Behaviour Prediction Factors. A Review of The Literature, Clin Exp Dent; 5(3): e150-3

[10] Zarone F, Russo S, Sorrentino R, 2011, From Porcelain-Fused-to-Metal to Zirconia: Clinical and Experimental Considerations, Dental Materials; 27: 83-96

[11] Kumar P, Vinitha B, Fathima G, Bone Graft in Dentistry, Journal of Pharmacy \& Bioallied Sciences; 5 (Suppl 1): S125-7

[12] Grageda E, Platelet-Rich Plasma and Bone Graft Materials: A Review and a Standardized Research Protocol, Implant Dent;13: 301-9 
[13] Marinescu A, Carligeriu V, Nica L, Onisei D, Rusu D, Boariu M, Epistatu D, Cioca D, Stratul S, 2010, Evaluation of Treatment Techniques for Advanced (Grade II-IV) Furcation Defects. A Literature Review of The Regenerative Methods, TMJ, 61 (No. 1 - 2): $115-24$
[14] Sheikh Z, Hamdan N, Glogauer M, 2017, Natural Graft Tissues and Synthetic Biomaterials for Periodontal and Alveolar Bone Reconstructive Applications: A Review, Biomaterials Research.; 21(9): 120 